\title{
Pre-sleep Arousal can be Associated with Efficient Processing of Sleep-related Information
}

Keisuke Takano ${ }^{a^{*}}$, Louise Vanden Poel ${ }^{b^{*}}$, and Filip Raes ${ }^{b}$

${ }^{a}$ Department of Psychology, Clinical Psychology and Psychotherapy,

Ludwig-Maximilians-University Munich, Germany;

${ }^{\mathrm{b}}$ Center for the Psychology of Learning and Experimental Psychopathology, University of Leuven, Belgium.

* These authors contributed to the current study equally; co-first authorship.

Correspondence concerning this article should be addressed to Keisuke Takano. Department of Psychology, Division of Clinical Psychology and Psychotherapy, Ludwig-Maximilians-University Munich, Leopoldstr.13, 80802 Munich, Germany.

Telephone: +49 8921805288 - Fax: +49 892180 5224. E-mail:

Keisuke.Takano@psy.lmu.de 


\begin{abstract}
Background and objectives: Cognitive bias to sleep-related information is thought to be a core feature of sleep disturbances. The bias may enhance pre-sleep arousal, such as excessive worry about sleeplessness, which prevents people from initiating normal sleep onset. The present study focused on (a) attention bias toward sleep-related stimuli and (b) difficulty in updating working memory for sleep-related stimuli as two possible mechanisms underlying pre-sleep cognitive arousal.
\end{abstract}

Method: Participants ( $n=61$, a community sample) completed a dot-probe task (with sleep-related and matched control word stimuli) and a 1-back and 2-back task (with sleep-related and non-sleep-related pictorial stimuli).

Results: For the dot-probe task, the results showed no significant association between pre-sleep cognitive arousal and sleep-related attention bias. However, the results of the 2-back task suggest that pre-sleep arousal is associated with decreased interference by sleep-related stimuli in maintaining non-sleep-related information. That is, individuals with higher levels of pre-sleep arousal are more efficient at processing sleep-related materials. Limitations: The non-clinical nature of the sample may limit the clinical implications of the findings.

Conclusions: Although the current results cannot be explained by the extant cognitive theories of insomnia, we offer an alternative explanation based on the idea of worry as mental habit: mental processes that occur frequently (e.g., repetitive thoughts about sleep) require less cognitive resource. Therefore, sleep-related information may be processed easily without consuming much cognitive effort.

Keywords; sleep, arousal, worry, attention bias, working memory 


\section{Introduction}

Cognitive models of insomnia (Espie, Broomfield, MacMahon, Macphee, \& Taylor, 2006; Harvey, 2002) have highlighted the role of excessive preoccupation with sleep in the development and maintenance of insomnia symptoms. Research has shown increased levels of worry and symptom-focused rumination (e.g., "If I cannot sleep well tonight, I will not be able to concentrate on my work tomorrow.") in individuals with sleep disturbances, compared to good sleepers (Gross \& Borkovec, 1982; Harvey, 2001; Thomsen, Mehlsen, Christensen \& Zachariae, 2003; Carney, Edinger, Meyer, Lindman, \& Istre, 2006). Such sleep- or insomnia-related cognition is particularly problematic when it occurs in pre-sleeping hours (e.g., Nicassio, Mendlowitz, Fussell, \& Petras, 1985), because thinking about sleep(lessness) and the possible consequences of poor sleep, along with general problem-solving and personal issues (self-focusing), are significant predictors of increased sleep latency (Takano, Sakamoto, \& Tanno, 2014; Wicklow \& Espie, 2000) and nighttime physiological arousal (Takano, Ueno, \& Tanno, 2014).

As a possible mechanism underlying excessive worry about sleep, researchers have investigated attention bias toward sleep-related information in individuals with sleep disturbances and clinical levels of insomnia. Studies have suggested that these individuals' attention is easily captured by and/or inefficiently disengaged from sleep-related stimuli (e.g., focusing on a clock to calculate how many hours they have slept; Woods, Marchetti, Biello, $\&$ Espie, 2009). One theory proposes that such attention bias could result in excessive monitoring of internal and external cues of sleep, which further triggers worry and rumination about sleeplessness and daytime dysfunctions (Harvey, 2002). Experimental studies have examined this sleep-related attention with various types of cognitive tasks, such as the dot-probe task, Posner task, and change blindness task (for a review, see Harris et al., 2015). Overall, the results support the presence of a sleep-related attentional bias in individuals with 
sleep disturbances (e.g., Jansson-Fröjmark, Bermås, \& Kjellén, 2012; Jones, Macphee, Broomfield, Jones, \& Espie, 2005; Marchetti, Biello, Broomfield, MacMahon, \& Espie, 2006; MacMahon, Broomfield, \& Espie, 2006; Woods et al., 2009).

However, sleep-related attention bias seems to be a more fragile phenomenon than initially expected, as more recent studies have failed to replicate attention bias in insomnia and non-clinical poor sleepers (e.g., Spiegelhalder et al., 2010, 2016). Two attention-bias-modification studies also failed to detect attention bias using the dot-probe task (Clarke, et al., 2016; Lancee, Yasiney, Brendel, Boffo, Clarke, \& Salemink, 2017). This is probably because "sleep-related stimuli" is a multi-faceted construct. Sleep-related stimuli were originally developed from an investigation of pre-sleep cognition (MacMahon et al., 2006; Wicklow \& Espie, 2000), which included both negatively valenced and emotionally neutral stimuli (e.g., tired, fatigue, dream, bed). Although even emotionally neutral sleep-related stimuli can be a target of selective attention (Harris et al., 2015), a recent Stroop study using "non-affective" sleep-related stimuli failed to detect significant differences between good and poor sleepers in response latency to sleep-related words (Barclay \& Ellis, 2013). This is a good strategy to control the effect of emotional valence, but the non-affective sleep-related stimuli do not cover "threatening signs" of insomnia (e.g., exhausted, aroused, restless). Thus, in the present study, we used the "original" set of sleep-related stimuli (e.g., MacMahon et al., 2006) with matched control stimuli in terms of valence and arousal in the dot-probe paradigm (one of the most widely used attention-bias tasks; cf. Bar-Haim, Lamy, Pergamin, Bakermans-Kranenburg, \& van Ijzendoorn, 2007). This stimulus set covers both emotionally negative and neutral materials reflecting the contents of pre-sleep cognitions (Taylor, Espie, \& White, 2003; Wicklow \& Espie, 2000), which allowed us to determine whether attention is specifically biased to sleep-related threatening information or merely the negative features of those stimuli. 
Although attention bias has been a target of many studies examining cognitive (dys)functions in sleep disturbances, other cognitive processes could also be relevant to pre-sleep worry and rumination. Given the persistent nature of insomnia-related cognition, difficulty in updating working memory (WM) is a candidate mechanism to explain the "stickiness" of sleep-related thinking. WM is a cognitive system that allows temporary storage and mental manipulation of information, which must balance two functions that are often in conflict with each other: maintenance and updating (Kessler \& Oberauer, 2014; Rac-Lubashevsky \& Kessler, 2016). The maintenance function refers to the limited storage capacity of WM, which keeps relevant information actively accessible. Moreover, it prevents the interference from irrelevant internal (e.g., long-term memory) and external (e.g., perceptual) input. The updating function refers to the ability to rapidly manipulate information held in WM when required, by adding new, relevant information and/or discarding information that is no longer relevant (Ecker, Oberauer, \& Lewandowsky, 2014; Ecker, Lewandowsky, \& Oberaurer, 2014).

Maintenance and updating are two conflicting demands, as they are indicators of stability versus flexibility, respectively. Computational models propose that there is a dynamic and selective input-gating mechanism that regulates the switching between these two functions (e.g., Frank, Loughry, \& O'Reilly, 2001; O’Reilly, 2006). When the gate is open, available information can enter WM, thereby allowing rapid updating. When the gate is closed, the current information in WM is maintained, while irrelevant information is prevented from entering. Evidence from previous research shows that switching between the WM functions of maintenance and updating, and therefore opening or closing the gate, results in an increased response time, or "switch cost" (Kessler \& Oberauer, 2014, 2015).

Specific biases and impairments in WM updating have been observed in individuals with depression and anxiety, which are also characterized by persistent cognition such as 
rumination and worry (e.g., Joormann \& Gotlib, 2008; Segal, Kessler, \& Anholt, 2015; Nolen-Hoeksema, Wisco, \& Lyubomirsky, 2008). Experimental studies using emotional n-back tasks, which measure the ability to maintain and update emotional information (e.g., happy and sad faces), have suggested that depressed individuals tend to be slower to disengage from sad stimuli and faster to disengage from happy stimuli in comparison to healthy controls (Levens \& Gotlib, 2010). Moreover, those individuals seem to have greater difficulty in removing irrelevant, negative information from WM and this interference is also associated with depressive rumination (Joormann \& Gotlib, 2008). Other studies suggest that rumination and worry are associated with general deficits in WM updating that are not limited to emotional processing (e.g., Meiran, Diamond, Toder, \& Nemets, 2017; Gustavson $\&$ Miyake, 2016). Given the potential role of persistent cognition in sleep disturbances (Espie et al., 2006; Harvey, 2002), it can be hypothesized that pre-sleep worry would be associated with an inability to maintain and update WM particularly for sleep-related information.

In summary, the present study tests the associations between pre-sleep worry and (a) attention bias to sleep-related stimuli, as measured by the dot-probe task, and (b) difficulty updating WM for sleep-related information, as measured by the n-back tasks. For attention bias, we had two (mutually exclusive) hypotheses. If people with higher levels of pre-sleep arousal show a greater attention bias to sleep-related stimuli, we could conclude that sleep-specific attention bias is independent of general emotional factors. However, if sleep-related attention bias is contaminated by emotional factors (and the bias can be attributed to vigilance to negatively valenced stimuli), the dot-probe performance should not correlate with pre-sleep arousal as our sleep-related and control stimuli are matched in valence and arousal. The second hypothesis was that individuals with higher (vs. lower) levels of pre-sleep arousal would significantly differ in their performances on the n-back (1-back and 2-back) tasks. In these tasks, participants are required to maintain and update 
WM in response to sequential presentation of sleep-related and non-sleep-related pictorial stimuli. Participants were asked to indicate whether the type (sleep-related or non-sleep-related) of stimulus of the current trial was the same as, or different than, that of the n-back trial. For the 1-back task, we predicted that individuals with higher levels of pre-sleep worry would show greater difficulty in removing sleep-related information from their WM. This persistency would be reflected in longer response times and lower accuracy for trials where participants had to switch from a sleep-related ( $n$ - 1 th trial) to a control stimulus ( $n$-th trial; i.e., switch cost). For the 2-back task, we predicted that individuals with higher levels of pre-sleep worry would show greater interference from a sleep-related stimulus on the $n$ - 1 th trial (reflected in longer response times and lower accuracy) when maintaining control stimuli between the $n$ - 2 and $n$-th trial.

\section{Method}

\subsection{Participants}

Sixty-one participants (50 women and 11 men; mean age $=22.2, S D=3.6$ years) were recruited from a large sample pool of a university, which covers its students and community living in the city and surrounds. There was no requirement for participation except that participants had to be fluent in Dutch. For their participation, participants received monetary compensation, either 10 or 20 euros, depending on their performance on a decision-making task (see also section 2.5 Procedure).

Although we did not have a good prior for an expected effect size of the n-back tasks, power analysis with G*power (Faul, Erdfelder, Lang, \& Buchner, 2007) suggested that the required sample size was $n=26$ - 59 to detect a correlation of $0.35-0.50$ under alpha $=0.05$ and beta $=0.80$. We assumed a moderate-to-large effect for the association between pre-sleep arousal and task performances (including the dot-probe and n-back tasks), because a recent review (Harris et al., 2015) suggested that the effect of the sleep-related attentional bias 
ranges from moderate to large sizes (e.g., $d=.74$ for attention bias measured by the dot-probe task, Jansson-Fröjmark et al., 2012).

\subsection{Measures}

\subsubsection{Pittsburgh Sleep Quality Index (PSQI; Buysse, Reynolds, Monk, Berman,}

\& Kupfer, 1989). The PSQI was used to assess subjective sleep disturbances over the past month. The PSQI is a self-report questionnaire consisting of 19 items that are grouped together into seven component scores: subjective sleep quality, sleep onset latency, sleep duration, habitual sleep efficiency, sleep disturbances, use of sleeping medication, and daytime dysfunction. Each score reflects experienced difficulty in that aspect of sleep, which is arranged to range from 0 (no difficulty) to 3 (severe difficulty). Summation of all component scores yields a global score. In the current study, the global score showed an acceptable level of internal consistency $(\alpha=.61)$.

2.2.2. Pre-Sleep Arousal Scale (PSAS; Nicassio et al., 1985). The PSAS assesses perceived cognitive and somatic arousal prior to falling asleep. This scale consists of 16 items, of which 8 items measure somatic arousal (e.g., dry feeling in mouth or throat) and the other 8 items measure cognitive arousal (e.g., worry about falling asleep). Participants indicate the extent to which they generally experience each item when trying to fall asleep on a 5-point scale $(1=$ not at all, $5=$ extremely $)$. In the current study, the PSAS had good internal consistency for both the cognitive $(\alpha=.77)$ and somatic arousal subscale $(\alpha=.84)$.

\subsection{Dot-probe task}

Attention bias to sleep-related stimuli was assessed using the dot-probe task. Each trial started with a fixation cross, displayed at the center of a computer screen for $500 \mathrm{~ms}$. This fixation display was followed by a word pair, which appeared on the left and right side of the screen (i.e., $5^{\circ}$ from the fixation to the center of the stimulus) for $500 \mathrm{~ms}$ (cf. Bar-Haim et al., 2007; however, see also Cooper \& Langton, 2006). The word pairs were either a 
combination of sleep-control or control-control words. The sleep words were adapted from previous studies (MacMahon, et al., 2006), for example bed, rest, and exhausted. The control words were selected from a large database of Dutch words (Moors et al., 2013) and were matched with the sleep words in length, valence, arousal, and frequency (see the Appendix). Immediately after the offset of the word stimuli, a target probe was presented in the place that one of the stimuli previously occupied. Participants were instructed to indicate the location of the probe by pressing the " 1 " (left) or " 3 " (right) key on a standard keyboard. After the participant's response, a fixation cross was presented for $1000 \mathrm{~ms}$. Participants performed four practice trials, followed by 160 main trials consisting of 40 congruent, 40 incongruent, and 80 neutral trials in a random order. In the congruent trials, the probe appeared on the same side of the sleep stimulus, whereas in the incongruent trials, the probe appeared on the opposite side of the sleep stimulus. In the neutral trials, only the control stimuli were presented on the screen. Attention bias score was calculated for each participant by subtracting the RT in the congruent trials from that in the incongruent trials.

\subsection{N-back tasks}

To measure the specific ability to update sleep-related information in WM, we designed a sleep-related n-back task, adapted from the emotional n-back tasks (Levens \& Gotlib, 2010; Pe, Koval, \& Kuppens, 2013). In a typical n-back task, participants are presented with a series of stimuli that are displayed sequentially on a computer screen. Participants are asked to indicate whether the stimulus of the current trial is the same as, or different than, that of the n-back trial. In the present study, we used sleep-related and non-sleep-related (i.e., garden-related) stimuli in the 1-back and 2-back paradigm (Figure 1). Both the 1-back and 2-back tasks consisted of 96 main trials, separated equally into four blocks (24 trials each). Before performing the main trials, participants practiced 24 trials that were not scored. On each trial, participants viewed a single image (either sleep-related or 
garden-related) that was presented centrally for $500 \mathrm{~ms}$, which was followed by a blank grey screen that lasted for $2500 \mathrm{~ms}$. They were instructed that they would see pictures in a sequential manner, and that the stimuli were objects that are typically found in a bed room (sleep-related) or in a garden (garden-related). In the 1-back task, participants matched the type ("sleep" or "garden") of stimuli between the current and 1-back trial by pressing the "1" key ("same") and the "3" key (“different"). Similarly, in the 2-back task, they matched the stimulus type between the current and 2-back trial. Participants were told that they may "lose their place" in the sequence of trials and forget which image was presented in the preceding trial. They were instructed, if this occurred, to start over- to view the current image and then start responding again from the second image on (Levens \& Gotlib, 2010).

We made four sets of pictorial stimuli: two were sleep-related, and the other two were garden-related images, which were used across both 1-back and 2-back tasks. Each of the "sleep" sets contained 12 grayscale images of different objects that can typically be found in a bedroom or are related to pre-sleep activities (i.e., alarm clock, baby crib, bathrobe, bed, blanket, hot water bottle, lamp, pajamas, pillow, slippers, teddy bear, and toothbrush). Both sets covered the same 12 categories of sleep-related objects, but images in the same object category were not perceptually identical (e.g., two different alarm clocks). Most of the object categories were adapted from a previous study of Jones et al. (2005), which also used sleep-related objects that are highly representative of a sleep environment.

Two sets of control (garden-related) stimuli were constructed using the same procedure of sleep-stimulus selection. A garden was assumed to be a "neutral" counterpart for a bedroom, as it is also a part of a house, but is not related to sleeping. Both sets of garden-related stimuli also covered 12 different object categories (i.e., animal, birdhouse, firewood, garden chair, gnome, child's toy, leaf, plant, rain boots, tool, tree, and tree fruit). Similar to the sleep-related stimuli, two perceptually different images for each object 
category were grouped into two different stimulus sets. In each block of the two n-back tasks (four blocks per task), we presented one set of sleep-related images and one set of garden-related images $^{1}$ (i.e., 24 stimuli in total).

In the 1-back task, there were four trial types in terms of the sequence of stimuli for $n-1$ and $n$-th trials: sleep-sleep, garden-garden, sleep-garden, and garden-sleep. The former two are "stay" trials, in which participants maintain the same stimulus type over the two consecutive trials; the latter two are "switch" trials, in which participants have to update their WM from a previous to a new stimulus type. It was expected that the "switch" trials would require a greater cost (increased RT and decreased accuracy) than the "stay" trials (Kessler \& Oberauer, 2014, 2015). Thus, the differences in RT and accuracy (a) between the sleep-garden and sleep-sleep trials, and (b) between the garden-sleep and garden-garden trials reflect cognitive costs to update WM (a) from sleep to garden, and (b) from garden to sleep representations, respectively. To balance the frequency of all four trial types, the order of stimulus presentation was quasi-randomized.

The 2-back task had eight possible trial sequences as combinations of sleep and garden stimuli for $n-2, n-1$, and $n$-th trials. We were specifically interested in the following four trial types: sleep-sleep-sleep, garden-garden-garden, sleep-garden-sleep, and garden-sleep-garden. In the former two "stay" trials, participants have to maintain a same (either sleep or garden) representation across the three consecutive trials. The latter two "interference" trials require participants to update WM in the $n$ - 1 th trial. The "odd" stimulus in this in-between trial interferes with the maintenance of a same representation from $n$ - 2 to $n$-th trial. Thus, the differences in RT and accuracy between the sleep-sleep-sleep and sleep-garden-sleep trial reflect the extent to which a "garden" stimulus interferes with

1 Prior to the experiment, a small group of students $(n=7)$, who were blinded to the purpose of the experiment, sorted the stimuli into sleep-related vs. garden-related categories. All the students sorted each image into the intended category, which assures that our stimuli do not have any ambiguity in interpreting them as being sleep-related or garden-related. 
maintaining a "sleep" representation, whereas the differences between garden-garden-garden and garden-sleep-garden trials specifically indicate the extent of interference by a "sleep" stimulus in "garden" processing. As with the 1-back task, all different trial types occurred at an almost equal frequency with a quasi-randomized presentation order.

\subsection{Procedure}

Participants were invited to the laboratory individually. Upon arrival, the procedure of the experiment was explained to them and they provided written informed consent. Subsequently, they received a bundle of questionnaires, including the PSQI and PSAS. After completing the questionnaires, participants performed three computer tasks: the dot-probe task, the n-back tasks, and the pay-per-view task. The present study was a part of a larger study concerning mental health and cognitive problems, which included other questionnaires and a cognitive task (i.e., the pay-per-view task) that are not reported here. Although the order of the three computer tasks was randomized across participants, the 1-back task was always performed before the 2-back task. Finally, participants were debriefed about the purpose of the experiment. All study protocols were approved by an institutional review board.

\section{Results}

Descriptive statistics and correlations of questionnaire measures are shown in Table 1. There were 28 participants with clinically significant levels of sleep disturbances that exceeded the cut-off score (> 5 on the PSQI). In line with a previous study (Nicassio et al., 1985), participants with higher levels of sleep disturbances (the PSQI scores) reported greater levels of pre-sleep arousal both on the somatic and cognitive subscales (Table 1). There were significant group differences between the high vs. low PSQI groups split by the cut-off, $t(45.9)=4.41$ for cognitive arousal and $t(32.5)=5.09$ for somatic arousal

\subsection{Dot-probe task}

Premature responses (RT < $200 \mathrm{~ms}, 52$ trials across all participants) were excluded 
from statistical analyses. Participants made correct responses for more than $99 \%$ of trials

(Table 2). The attention bias score (i.e., difference in RT between the congruent vs.

incongruent condition) was not significantly correlated with sleep measures, the PSQI, and PSAS (Table 3). These null correlations suggest that attention bias to sleep-related stimuli cannot be observed by the controlled stimulus set, where sleep-related and non-sleep-related words are matched in valence and arousal.

\subsection{N-back tasks}

Before performing specific statistical analyses, we eliminated premature responses (RT $<200 \mathrm{~ms}$; 4 trials in the 1-back and 6 trials in the 2-back task across all participants). Furthermore, the data of 14 participants were excluded from analyses for the following reasons: responses of three participants were not recorded because of device malfunction, and the remaining 11 participants did not respond for the majority of trials (> 50\%) in either the 1-back $(n=2)$, 2-back $(n=6)$, or both tasks $(n=3)$. These participants typically used a specific response strategy (e.g., responding only once every three trials in the 2-back task), which was considered an unreliable measure of WM functions.

The mean accuracy and RT are shown in Table 3. As preliminary analyses, we performed two-way ANOVAs on accuracy and RT of the 1-back task with stimulus type ("sleep" vs. "garden" at $n$-1th trial) and trial type ("switch" vs. "stay") both as within-person factors. The ANOVA on accuracy revealed significant main effects of the stimulus type, $F(1,46)=19.28, p<.001, \eta_{\mathrm{p}}{ }^{2}=0.30$, and the trial type, $F(1,46)=6.69, p=.01, \eta_{\mathrm{p}}{ }^{2}=0.13$, but no significant interaction between the stimulus type and trial type, $F(1,46)=2.39, p=.13$, $\eta_{\mathrm{p}}{ }^{2}=0.05$. The ANOVA on RT showed a similar result pattern: significant main effects of the stimulus type, $F(1,46)=20.41, p<.001, \eta_{\mathrm{p}}{ }^{2}=0.31$, and the trial type, $F(1,46)=48.60, p$

$<.001, \eta_{\mathrm{p}}{ }^{2}=0.51$, and a significant interaction between the stimulus type and trial type, $F(1,46)=17.17, p<.001, \eta_{\mathrm{p}}^{2}=0.27$. This significant interaction was due to a greater 
difference between garden-sleep vs. garden-garden trials, $t(46)=6.98, d_{z}=1.02$, than that between sleep-garden vs. sleep-sleep trials, $t(47)=3.86, d_{z}=0.56$. Overall these results suggest that (a) the "switch" trials are more difficult than the "stay" trials in term of accuracy and RT, and that (b) switching from garden to sleep takes longer than switching from sleep to garden.

For the 2-back task, similar two-way ANOVAs were performed on accuracy and RT with the trial type (stay vs. interference) and stimulus type (sleep vs. garden, at $n$ - 2 th trial) both as within-person factors. For response accuracy, the ANOVA showed a significant main effect of the trial type, $F(1,46)=10.75, p<.001, \eta_{\mathrm{p}}{ }^{2}=0.19$, but no significant main effect of the stimulus type, $F(1,46)=1.38, p=.25, \eta_{\mathrm{p}}{ }^{2}=0.03$, or interaction, $F(1,46)=2.72, p=.11$, $\eta_{\mathrm{p}}{ }^{2}=0.06$. The ANOVA on RT showed similar results: a significant main effect was found for trial type, $F(1,46)=21.27, p<.001, \eta_{\mathrm{p}}{ }^{2}=0.32$, but not for the stimulus type, $F(1,46)=1.24$, $p=.27, \eta_{\mathrm{p}}{ }^{2}=0.03$, or interaction, $F(1,46)=0.47, p=.50, \eta_{\mathrm{p}}{ }^{2}=0.01$. These results suggest that participants' responses are interfered with by an "odd" stimulus at $n$ - 1 th trial while maintaining a same stimulus representation between $n$ - 2 and $n$-th trials.

Correlational analysis (see Table 3) showed no significant associations between the sleep measures and switch costs (i.e., differences between the switch vs. stay trials) in the 1-back task. However, in the 2-back task, the sleep-interference score in RT (i.e., difference between the garden-sleep-garden vs. garden-garden-garden trials) was negatively correlated with the measures of pre-sleep cognitive $(r=-0.33, p=0.025)$ and somatic arousal $(r=-0.39$, $p=0.006$ ). The base response speed (RT in GGG trials) was not significantly correlated with the PSAS measures $(r \mathrm{~s}<.07, p \mathrm{~s}>.63)$. Although the directions of the correlations were opposite to our hypothesis (i.e., arousal factors are associated with increased interference by sleep stimuli), these results suggest that participants with higher levels of pre-sleep arousal experience less interference from sleep-related stimuli in processing/maintaining 
non-sleep-related information. This can be viewed as a lack of normative interference (Figure 2), as people without pre-sleep arousal typically had an interference effect (longer RT in the “interference" relative to "stay" trials). If we correct the alpha level for multiple tests (four 2-back parameters * two pre-sleep arousal measures), the Bonferroni-corrected alpha is 0.05 / $8=0.0062$. Under this criterion, the correlation between the sleep-interference score and somatic arousal remained statistically significant.

Note that none of the accuracy scores were significantly correlated with the sleep and arousal measures (Table 3). This inconsistency is likely because most of the errors observed in the current study were commission errors (74\%; participants pressed an incorrect button). Therefore, omission errors (participants incorrectly did not press a button) were less frequent, although the number of omission errors, but not commission errors, is known to correlate with RT measures in WM tasks (Muel, 2017). The RT and accuracy indices may reflect different phenomena, with RT reflecting a delay in updating WM and accuracy indicating a response inhibition or influence of false memory.

\section{Discussion}

The present study examined whether participants with higher (vs. lower) levels of pre-sleep arousal, particularly for the cognitive component (i.e., worry), differ in (a) attention bias toward sleep-related information and (b) the ability to update sleep-related stimuli in WM. In accordance with cognitive models of insomnia (Espie et al., 2006; Harvey, 2002), we hypothesized that pre-sleep arousal would be associated with attention bias to sleep-related stimuli and an impaired ability to update WM. The results, however, showed that pre-sleep arousal is not associated with attention bias as measured by the dot-probe task. Furthermore, no significant association was found between pre-sleep arousal and inflexibility to update WM (i.e., switching cost) in the 1-back task with sleep-related and control (garden-related) stimuli. Although these findings suggest that neither attention bias nor difficulty in removing 
sleep-related information from WM is relevant to pre-sleep arousal, the results of the 2-back task showed a significant negative association between pre-sleep arousal and interference by sleep-related stimuli when maintaining non-sleep-related information. Although this negative association is opposite to our prediction, it implies that rather than experiencing more interference by (and less efficient in inhibiting) an irrelevant sleep-related stimulus, participants with higher levels of pre-sleep arousal seem to experience less interference by (and more efficient in inhibiting) a sleep-related distractor when processing non-sleep-related information.

Attention bias to sleep-related stimuli has been a topic in the insomnia-cognition literature (e.g., Espie et al., 2006; Harvey 2002). However, recent studies call into question whether attentional bias is actually a core feature of insomnia (e.g., Spiegelhalder et al., 2016). In the present study, we used matched control stimuli to eliminate potential contamination by emotional (negative valence and arousal) factors. In line with the null findings of Barclay and Ellis (2013) with "non-affective" sleep-related stimuli, we found no significant associations between the attention bias score and self-report sleep measures. These results may suggest that attention bias in sleep disturbances is more influenced by emotional factors (negative valence) than by the sleep-relatedness of the stimuli (i.e., content factor). Another possible explanation for this failure to detect sleep-related attention bias is that the bias score of the dot-probe task is sometimes not stable to parameterize individual differences in attention functioning. Recent studies claim that the attention bias score has limited reliability for internal consistency and test-retest reliability (with negative stimuli: Kappenman, MacNamara, \& Proudfit, 2015; Schmukle, 2006; Van Bockstaele, Verschuere, Koster, Tibboel, De Houwer, \& Crombez, 2011; with sleep-related stimuli: Lancee et al., 2017). In line with these arguments, we found a low split-half reliability for the attention bias score, $r=0.09$, in the current study. Although there is at least one dot-probe study that 
reported acceptable reliability for the bias score (Bar-Haim et al., 2010), this low-reliability issue is becoming widely recognized (Van Bockstaele et al., 2014) and poses a concern in the replicability and reproducibility of the attention-bias findings ${ }^{2}$. One might think that the non-clinical nature of our sample could also explain the absence of attention bias. Indeed, most of the studies that found significant sleep-related attention bias examined people with primary insomnia (Woods et al., 2009; MacMahon et al., 2006), although some other "clinical" studies failed to detect attention bias (Lancee et al., 2017; Spiegelhalder et al., 2016). More importantly, the reliability issue of the dot-probe task was observed across different experimental settings, such as clinical vs. nonclinical samples and word vs. pictorial stimuli (for a review, see Rodebaugh et al., 2016). This poor psychometric property of the task would, therefore, lie behind the replication failures as a more fundamental and common issues in the field.

While extant studies on sleep-related cognition have mainly focused on attention functioning (Harris et al., 2015; Jansson-Fröjmark et al., 2013; Woods et al., 2009), the present study is among the first to examine whether a sleep-related bias is present in other cognitive domains, i.e., WM updating. Along with the literature of WM updating and persistent negative cognition (Joormann \& Gotlib, 2008; Levens \& Gotlib, 2010), we predicted that individuals with pre-sleep worry would show slower disengagement from sleep-related stimuli and greater difficulty in keeping irrelevant (sleep-related) information from entering WM. However, the results of the 2-back task suggest the opposite pattern for the association, that is, pre-sleep arousal is associated with less interference from sleep-related stimuli. Interestingly, both the cognitive and somatic arousal measures seem to be correlated with this decreased interference effect. More rigorous tests with a corrected

\footnotetext{
${ }^{2}$ New attention-bias indices are proposed to resolve the reliability issue (Zvielli et al., 2014, 2016). Although some of them demonstrated a good reliability (Rodebaugh et al., 2016), the new indices are not recommended to use for empirical purposes due to the inability to uncouple measurement error from true bias scores (Kruijt, Field, \& Fox, 2016).
} 
alpha for multiple tests suggested that the correlation with somatic, but not cognitive, arousal is reliably associated with the interference effect. This may imply that the efficient processing is more relevant to increased somatic arousal, which in general influences attention and memory systems (e.g., Sharot \& Phelps, 2004). However, we do not see substantial differences in the magnitude of the correlations ( $r \mathrm{~s}=0.31$ vs. 0.39 ), and these two types of arousal are known to be strongly associated (e.g., Nicassio et al., 1985). Worry enhances physiological arousal (Pieper, Brosschot, van der Leeden, \& Thayer, 2010) and perceiving autonomic arousal may further fuel anxiety and worry. The current study is not equipped to dissociate the effects of cognitive and somatic arousal, but it would be interesting to employ specific manipulations to induce cognitive (e.g., Wuyts et al., 2012) and somatic arousal (e.g., Tang \& Harvey, 2004) in future research.

Although our findings do not fit the extant cognitive theories of insomnia (Espie et al., 2006; Harvey, 2002), an alternative explanation can be found in the literature of depressive rumination and anxious worry. Van Lier, Vervliet, and Raes (2015) used a dual task paradigm to test if an induced maladaptive (vs. adaptive) thinking style influences WM performance. They found that the maladaptive (or abstract ruminative) thinking does not interfere with WM functions for individuals with depressive symptoms, suggesting that the maladaptive thinking has been automated in depressed individuals and thus consumes less WM capacity. Hertel (2004) considered persistent cognition as a mental habit, a process that occurs without conscious awareness, effort, and mental efficiency (Bargh, 1994). The last dimension, mental efficiency, presumes that a habitual mental process tends to require relatively low cognitive resources. Such habitual negative thinking is more resistant to change, because it is often out of conscious control (Watkins \& Nolen-Hoeksema, 2014). This "mental habit" view of persistent cognition could explain our findings that individuals with higher levels of pre-sleep worry experience less interference from sleep-related stimuli. 
Given that these individuals frequently tend to worry and ruminate about their sleep(lessness) (Harvey, 2002), this pre-occupation with sleep may eventually become a mental habit that does not require extensive cognitive resource when processing sleep-related information.

There are some limitations to consider in the present study. First, we did not control the saliency of the pictorial stimuli in the n-back tasks. This might influence the task performances, particularly for the 1-back task, where we observed significantly longer RTs in "garden" trials than "sleep" trials. Another important issue in the n-back task is that a substantial number of participants could not perform the 2-back task with sufficient accuracy (> 50\%). Although we did not prevent participants from using a specific response strategy (i.e., responding every three trials) that caused extremely low response accuracy, such a specific response strategy should not have been mentioned in the instructions (cf. Levens \& Gotlib, 2010). Third, the current 2-back task cannot capture the "n+1 lure" effect (e.g., Szmalec, Verbruggen, Vandierendonck, \& Kemps, 2011), which may be also relevant to perseverative cognition (or difficulty in eliminating unnecessary information from WM). It is known that responses are interfered with when a newly presented item is different from the n-back item but same to one of its neighbors (e.g., A-B-C-A, where the current stimulus is same to that at the n-3 back trial). This interference effect cannot be formulated in our n-back tasks because there were only two stimulus types (sleep vs. non-sleep). Given that similar “n+1" effects with emotional stimuli were observed in depression (Levens \& Gotlib, 2010), future research needs to clarify if this response interference would take place in the context of sleep and insomnia. Fourth, most of our participants were female university students, which may limit the generalizability of our findings. Given that women are more vulnerable to sleep problems than men (e.g., Zhang \& Wing, 2006), future research needs to examine whether our results reflect a gender-specific phenomenon.

Nevertheless, our findings, particularly of the 2-back task, open a novel perspective 
that people with higher levels of pre-sleep arousal have a better ability to process sleep-related stimuli. This finding could imply that frequent worry and rumination about sleep eventually becomes a mental habit that no longer requires extensive cognitive recourse to process sleep-related information. Although the non-clinical nature of our sample may limit the clinical impact of our findings, there was a high prevalence of participants who reported clinically significant levels of sleep disturbances $(46 \%$; above the cut-off of the PSQI, > 5) in the present study. The fact that many of the participants were university students may partly explain the high prevalence of significant sleep problems (Lund, Reider, Whiting, \& Prichard, 2010). This echoes the importance of investigating sleep problems in a young population, but further research in clinical samples is still warranted to gain more insight into cognitive processes in people with insomnia and sleep disorders. 
Acknowledgement

Keisuke Takano was supported by the Alexander von Humboldt Foundation Postdoctoral Research Fellowship. Filip Raes was supported by the KU Leuven Research Council grant PF/10/005. We thank Sarah Van den Brande for her assistance in data collection. 


\section{References}

Bar-Haim, Y., Holoshitz, Y., Eldar, S., Frenkel, T. I., Muller, D., Charney, D. S., ... Wald, I. (2010). Life-threatening danger and suppression of attention bias to threat. American Journal of Psychiatry, 167, 694-688. doi: 10.1176/appi.ajp.2009.09070956

Bar-Haim, Y., Lamy, D., Pergamin, L., Bakermans-Kranenburg, M. J., \& van Ijzendoorn, M. H. (2007).Threat-related attentional bias in anxious and nonanxious individuals: a meta-analytic study. Psychological Bulletin, 133, 1-12. doi:

10.1037/0033-2909.133.1.1

Barclay, N. L. \& Ellis, J. G. (2013). Sleep-related attentional bias in poor versus good sleepers is independent of affective valence. Journal of Sleep Research, 22, 414-421. doi: $10.1111 /$ jsr. 12035 .

Bargh, J. A. (1994). The four horsemen of automaticity: Awareness, intention, efficiency, and control in social cognition. In R.S. Wyer \& T. K. Srull (Eds.), Handbook of social cognition (pp. 1-40). Hillsdale, NJ: Erlbaum.

Buysse, D. J., Reynolds, C. F., Monk, T. H., Berman, S. R., \& Kupfer, D. J. (1989). The Pittsburgh sleep quality index: A new instrument for psychiatric practice and research. Psychiatry Research, 28, 1190-1204. doi:10.1016/0165-1781(89)90047-4

Carney, C. E., Edinger, J. D., Meyer, B., Lindman, L., \& Istre, T. (2006). Symptom-focused rumination and sleep disturbance. Behavioral Sleep Medicine, 4, 228-241. doi:10.1207/s15402010bsm0404_3

Clarke, P. J., Bedford, K., Notebaert, L., Bucks, R. S., Rudaizky, D., Milkins, B. C., MacLeod, C. (2016). Assessing the therapeutic potential of targeted attentional bias modification for insomnia using smartphone delivery. Psychotherapy and Psychosomatics, 85, 187-189. doi: 10.1159/000442025

Cooper, R. M., \& Langton, S. R. (2006). Attentional bias to angry faces using the dot-probe 
task? It depends when you look for it. Behaviour Research and Therapy, 44, 1321-1329. doi: 10.1016/j.brat.2005.10.004

Ecker, U., Lewandowsky, S., \& Oberauer, K. (2014). Removal of information from working memory: A specific updating process. Journal of Memory and Language, 74, 77-90. doi:10.1016/j.jml.2013.09.003

Ecker, U., Oberauer, K., \& Lewandowsky, S. (2014). Working memory updating involves item specific removal. Journal of Memory and Language, 74, 1-15. doi:10.1016/j.jml.2014.03.006

Espie, C. A., Broomfield, N. M., MacMahon, K. M. A., Macphee, L. M., \& Taylor, L. M. (2006). The attention-intention-effort pathway in the development of psychophysiologic insomnia: A theoretical review. Sleep Medicine Reviews, 10, 215245. doi:10.1016/j.smrv.2006.03.002

Faul, F., Erdfelder, E., Lang, A., \& Buchner, A. (2007). G*Power 3: A flexible statistical power analysis program for the social, behavioral, and biomedical sciences. Behavior Research Methods, 39, 175-191. doi: 10.3758/BF03193146

Gross, R. T., \& Borkovec, T. D. (1982). Effects of cognitive intrusion manipulation on the sleep-onset latency of good sleepers. Behavior Therapy, 13, 112-116. doi:10.1016/S0005-7894(82)80054-3

Gustavson, D. E., \& Miyake, A. (2016). Trait worry is associated with difficulties in working memory updating. Cognition and Emotion, 30, 12891303.doi:10.1080/02699931.2015.1060194

Harris, K., Spiegelhalder, K., Espie, C. A., MacMahon, K. M., Woods, H. C., \& Kyle, S. D. (2015). Sleep-related attentional bias in insomnia: A state-of-the-science review. Clinical Psychology Review, 42, 16-27. doi: 10.1016/j.cpr.2015.08.001.

Harvey, A. G. (2002). A cognitive model of insomnia. Behaviour Research and Therapy, 40, 
869-893. doi:10.1016/S0005-7967(01)00061-4

Hertel, P. T. (2004). Memory for emotional and non-emotional events in depression: A question of habit? In D. Reisberg \& P. Hertel (Eds.), Memory and emotion (pp. 186216). New York: Oxford University Press.

doi:10.1093/acprof:oso/9780195158564.003.0006

Jansson-Fröjmark, M., Bermås, M., \& Kjellén, A. (2013). Attentional bias in insomnia: The dot-probe task with pictorial stimuli depicting daytime fatigue/malaise. Cognitive Therapy and Research, 37, 534-546. doi:10.1007/s10608-012-9486-z

Jones, B. T., Macphee, L. M., Broomfield, N. M., Jones, B. C., \& Espie, C. A. (2005). Sleep-related attentional bias in good, moderate, and poor (primary insomnia) sleepers. Journal of Abnormal Psychology, 114, 249-258. doi:10.1037/0021-843X.114.2.249

Joormann, J., \& Gotlib, I. H. (2008). Updating the contents of working memory in depression: Interference from irrelevant negative material. Journal of Abnormal Psychology, 117, 182-192. doi: 10.1037/0021-843X.117.1.182

Kappenman, E. S., MacNamara, A., Proudfit, G. H. (2015). Electrocortical evidence for rapid allocation of attention to threat in the dot-probe task. Social Cognitive Affective Neuroscience, 10, 577-583. doi: 10.1093/scan/nsu098

Kessler, Y., \& Oberauer, K. (2014). Working memory updating latency reflects the cost of switching between maintenance and updating modes of operation. Journal of Experimental Psychology: Learning, Memory, and Cognition, 40, 738-754. doi: $10.1037 / \mathrm{a} 0035545$

Kessler, Y., \& Oberauer, K. (2015). Forward scanning in verbal working memory updating. Psychonomic Bulletin \& Review, 22, 1770-1776. doi:.3758/s13423-015-0853-0

Lancee, J., Yasiney, S. L., Brendel, R. S., Boffo, M., Clarke, P. J. F., Salemink, E. (2017). Attentional bias modification training for insomnia: A double-blind placebo controlled 
randomized trial. PLoS One, 12, e0174531. doi: 10.1371/journal.pone.0174531.

Levens, S. M., \& Gotlib, I. H. (2010). Updating positive and negative stimuli in working memory in depression. Journal of Experimental Psychology: General, 139, 654-664. doi:10.1037/a0020283

Lund, H. G., Reider, B. D., Whiting, A. B., \& Prichard, J. R. (2010). Sleep patterns and predictors of disturbed sleep in a large population of college students. Journal of Adolescent Health, 46, 124-132. doi: 10.1016/j.jadohealth.2009.06.016

MacMahon, K. M., Broomfield, N. M., \& Espie, C. A. (2006). Attention bias for sleep-related stimuli in primary insomnia and delayed sleep phase syndrome using the dot-probe task. Sleep, 29, 1420-1427.

Marchetti, L. M., Biello, S. M., Broomfield, N. M., MacMahon, K. M., \& Espie, C. A. (2006). Who is pre-occupied with sleep? A comparison of attention bias in people with psychophysiological insomnia, delayed sleep phase syndrome and good sleepers using the induced change blindness paradigm. Journal of Sleep Research, 15, 212-221. doi:10.1111/j.1365-2869.2006.00510.x

Meiran, N., Diamond, G. M., Toder, D., \& Nemets, B. (2017). Cognitive rigidity in unipolar depression and obsessive compulsive disorder: Examination of task switching, Stroop, working memory updating and post-conflict adaptation. Psychiatry Research, 185, 149-156. doi:10.1016/j.psychres.2010.04.044

Moors, A., De Houwer, J., Hermans, D., Wanmaker, S., van Schie, K., Van Harmelen, A. L., ... Brysbaert, M. (2013). Norms of valence, arousal, dominance, and age of acquisition for 4,300 Dutch words. Behavior Research Methods, 45, 169-177. doi: 10.3758/s13428-012-0243-8.

Meule, A. (2017). Reporting and Interpreting Working Memory Performance in n-back Tasks. Frontiers in Psychology, 8, 352, doi: 10.3389/fpsyg.2017.00352. 
Nicassio, P. M., Mendlowitz, D. R., Fussell, J. J., \& Petras, L. (1985). The phenomenology of the pre-sleep state: The development of the pre-sleep arousal scale. Behavioral Research and Therapy, 23, 263-271. doi:10.1016/0005-7967(85)90004-X

Nolen-Hoeksema, S., Wisco, B. E., \& Lyubomirsky, S. (2007). Rethinking rumination. Perspectives on Psychological Science, 3, 400-424. doi: 10.1111/j.1745-6924.2008.00088.x.

Pe, M. L., Koval, P., \& Kuppens, P. (2013). Executive well-being: updating of positive stimuli in working memory is associated with subjective well-being. Cognition, 126, 335-340. doi: 10.1016/j.cognition.2012.10.002.

Pieper, S., Brosschot, J. F., van der Leeden, R., \& Thayer, J. F. (2010). Prolonged cardiac effects of momentary assessed stressful events and worry episodes. Psychosomatic Medicine, 72, 570-577. doi: 10.1097/PSY.0b013e3181dbc0e9

Rac-Lubashevsky, R., \& Kessler, Y. (2016). Decomposing the n-back task: An individual differences study using the reference-back paradigm. Neuropsychologia, 90, 190-199. doi:10.1016/j.neuropsychologia.2016.07.013

Rodebaugh, T. L., Scullin, R. B., Langer, J. K., Dixon, D. J., Huppert, J. D., Bernstein, A., ... \& Lenze, E. J. (2016). Unreliability as a threat to understanding psychopathology: The cautionary tale of attentional bias. Journal of Abnormal Psychology, 125, 840-851. doi: 10.1037/abn0000184

Schmukle, S. C. (2005). Unreliability of the dot probe task. European Journal of Personality, 19, 595-605. doi: 10.1002/per.554

Segal, A., Kessler, Y., \& Anholt, G. E. (2015). Updating the emotional content of working memory in social anxiety. Journal of Behavior Therapy and Experimental Psychiatry, 48, 110-117. doi:10.1016/j.jbtep.2015.02.012 
Sharot, T., \& Phelps, E. A. (2004). How arousal modulates memory: disentangling the effects of attention and retention. Cognitive, Affective, \& Behavioral Neuroscience, 4(3), 294306. doi: 10.3758/CABN.4.3.29

Spiegelhalder, K., Kyle, S. D., Feige, B., Prem, M., Nissen, C., Espie, C. A., \& Riemann, D. (2010). The impact of sleep-related attentional bias on polysomnographically measured sleep in primary insomnia. Sleep, 33, 107-112.

Spiegelhalder, K., Baglioni, C., Regen, W., Kyle, S. D., Nissen, C., Hennig, J., ... Riemann, D. (2016). Brain Reactivity and Selective Attention to Sleep-Related Words in Patients With Chronic Insomnia. Behavioral Sleep Medicine, 14, 1-15. doi: 10.1080/15402002.2016.1253014.

Szmalec, A., Verbruggen, F., Vandierendonck, A., \& Kemps, E. (2011). Control of interference during working memory updating. Journal of Experimental Psychology: Human Perception and Performance. 37(1), 137-151. doi: 10.1037/a0020365.

Takano, K., Sakamoto, S., \& Tanno, Y. (2014). Repetitive thought impairs sleep quality: an experience sampling study. Behavior Therapy, 45, 67-82. doi: 10.1016/j.beth.2013.09.004.

Takano, K., Ueno, M., \& Tanno, Y. (2014). Self-focused thinking predicts nighttime physiological de-arousal. Biological Psychology, 97, 9-14. doi: 10.1016/j.biopsycho.2014.01.001.

Tang, N. K. Y., \& Harvey, A. G. (2004). Effects of Cognitive Arousal and Physiological Arousal on Sleep Perception. Sleep, 27, 69-78.

Taylor, L. M., Espie, C. A., \& White, C. A. (2003). Attentional bias in people with acute versus persistent insomnia secondary to cancer. Behavioral Sleep Medicine, 1, 200-212. doi: 10.1207/S15402010BSM0104_3 
Thomsen, D. K., Mehlsen, M. Y., Christensen, S., \& Zachariae, R. (2003). Rumination relationship with negative mood and sleep quality. Personality and Individual Differences, 34, 1293-1301. doi:10.1016/S0191-8869(02)00120-4

Van Bockstaele, B., Verschuere, B., Koster, E. H. W., Tibboel, H., De Houwer, J., Crombez, G. (2011). Differential predictive power of self report and implicit measures on behavioural and physiological fear responses to spiders. International Journal of Psychophysiology, 79, 166-174. doi: 10.1016/j.ijpsycho.2010.10.003

Van Bockstaele, B., Verschuere, B., Tibboel, H., De Houwer, J., Crombez, G., \& Koster E. H. W. (2014). A review of current evidence for the causal impact of attentional bias on fear and anxiety. Psychological Bulletin, 140, 682-721. doi: 10.1037/a0034834.

Van Lier, J., Vervliet, B., \& Raes, F. (2015). The effect of abstract 'why' thoughts on working memory. Paper presented at the Rome Workshop on Experimental Psychopathology. Rome, Italy, 20-21 March 2015.

Wicklow, A., \& Espie, C. A. (2000). Intrusive thoughts and their relationship to actigraphic measurement of sleep: towards a cognitive model of insomnia. Behaviour Research and Therapy, 38, 679-93.

Woods, H., Marchetti, L. M., Biello, S. M., \& Espie, C. A. (2009). The clock as a focus of selective attention in those with primary insomnia: An experimental study using a modified Posner paradigm. Behaviour Research and Therapy, 47, 231-236. doi:10.1016/j.brat.2008.12.009

Wuyts, J., De Valck, E., Vandekerckhove, M., Pattyn, N., Bulckaert, A., Berckmans, D., ... Cluydts, R. (2012). The influence of pre-sleep cognitive arousal on sleep onset processes. International Journal Psychophysiology, 83(1), 8-15. doi: 10.1016/j.ijpsycho.2011.09.016

Zvielli, A., Vrijsen, J. N., Koster, E. H., \& Bernstein, A. (2016). Attentional bias temporal 
dynamics in remitted depression. Journal of Abnormal Psychology, 125, 768-776. doi: 10.1037/abn0000190.

Zvielli, A., Bernstein, A., \& Koster, E. H. W. (2015). Temporal dynamics of attention bias. Clinical Psychological Science, 3, 772-788. doi.org/10.1177/2167702614551572 
Table 1.

Descriptive Statistics and Correlations Between Measures of Sleep and Pre-sleep arousal (N $=61)$

\begin{tabular}{|l|c|c|c|c|}
\hline \multirow{2}{*}{ Measure } & \multirow{2}{*}{$M$} & SD & \multicolumn{2}{c|}{ Correlations with } \\
\cline { 3 - 5 } & & & PSQI & PSAS Cog \\
\hline PSQI & 5.64 & 2.44 & - & \\
\hline PSAS Cog & 19.74 & 6.14 & $.61^{*}$ & - \\
\hline PSAS Som & 12.43 & 4.48 & $.61^{*}$ & $.43^{*}$ \\
\hline
\end{tabular}

Note. PSQI = Pittsburgh Sleep Quality Index; PSAS Cog / Som = Pre-sleep Arousal Scale Cognitive / Somatic subscale. $* p<.01$. 
Table 2

Means (and SD) of Task Performances on the Dot-probe and N-back Tasks

\begin{tabular}{|c|c|c|}
\hline Task and Trial types & Accuracy & Response time (ms) \\
\hline \multicolumn{3}{|l|}{ Dot-probe task } \\
\hline Congruent & $0.99(0.02)$ & $408.3(56.2)$ \\
\hline Incongruent & $0.99(0.02)$ & $406.9(52.1)$ \\
\hline Neutral & $0.99(0.02)$ & $409.2(55.0)$ \\
\hline \multicolumn{3}{|l|}{ 1-back task } \\
\hline GG & $0.84(0.18)$ & $789.1(193.4)$ \\
\hline GS & $0.79(0.20)$ & $893.9(231.4)$ \\
\hline SS & $0.93(0.10)$ & 764.6 (170.7) \\
\hline SG & $0.91(0.08)$ & 805.4 (190.9) \\
\hline \multicolumn{3}{|l|}{ 2-back task } \\
\hline GGG & $0.87(0.13)$ & $836.9(244.1)$ \\
\hline GSG & $0.75(0.23)$ & $938.8(245.7)$ \\
\hline SSS & $0.85(0.15)$ & $830.4(228.2)$ \\
\hline SGS & $0.81(0.19)$ & $912.2(260.8)$ \\
\hline
\end{tabular}

Note. $\mathrm{G}=$ garden-related stimulus, $\mathrm{S}$ = sleep-related stimulus; $\mathrm{GS}$ = a garden-related stimulus at the $n$ - 1 th trial, followed by a sleep-related stimulus at the $n$-th trial. $N=61$ for the dot-probe task; $N=47$ for the n-back tasks. 
Table 3

Correlations between Task Performance on the Cognitive Tasks and Sleep Measures

\begin{tabular}{|c|c|c|c|c|}
\hline Tasks & Indices & PSQI & $\begin{array}{c}\text { PSAS } \\
\text { Cognitive } \\
\text { arousal }\end{array}$ & $\begin{array}{c}\text { PSAS } \\
\text { Somatic } \\
\text { arousal }\end{array}$ \\
\hline Dot-probe & Bias score (Incongruent - Congruent) & .04 & -.01 & .11 \\
\hline \multirow[t]{6}{*}{ 1-back } & Accuracy & & & \\
\hline & Switch to Sleep (GS - GG) & .10 & -.06 & .26 \\
\hline & Switch to Garden $(\mathrm{SG}-\mathrm{SS})$ & .28 & .12 & .05 \\
\hline & Response time & & & \\
\hline & Switch to Sleep (GS - GG) & -.04 & .00 & -.14 \\
\hline & Switch to Garden $(\mathrm{SG}-\mathrm{SS})$ & .03 & -.17 & -.04 \\
\hline \multirow[t]{6}{*}{ 2-back } & Accuracy & & & \\
\hline & Interference by Sleep (GSG - GGG) & .05 & -.08 & .04 \\
\hline & Interference by Garden (SGS - SSS) & .12 & .06 & .07 \\
\hline & Response time & & & \\
\hline & Interference by Sleep (GSG - GGG) & -.26 & $-.33 *$ & $-.39 * *$ \\
\hline & Interference by Garden (SGS - SSS) & .27 & .11 & .10 \\
\hline
\end{tabular}

Note. PSQI = Pittsburgh Sleep Quality Index; PSAS = Pre-sleep Arousal Scale. G = Garden stimulus; $\mathrm{S}=$ Sleep stimulus. ${ }^{* *} p<.01, * p<.05 . N=61$ for the dot-probe task; $N=47$ for the n-back tasks. 
(A) The 1-back task

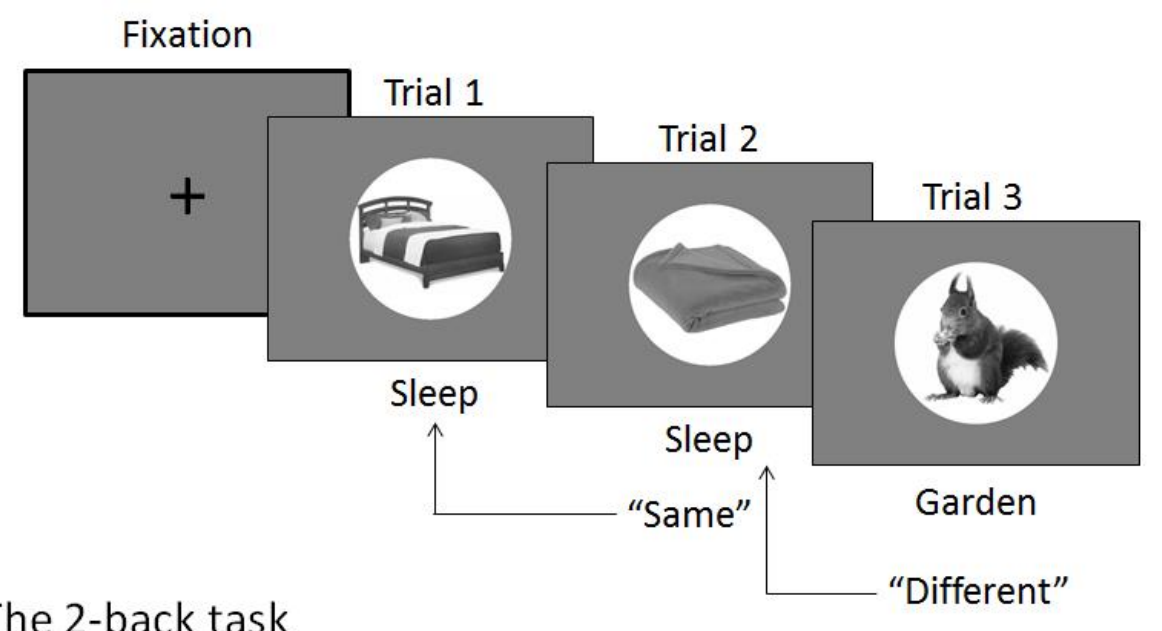

(B) The 2-back task

Fixation

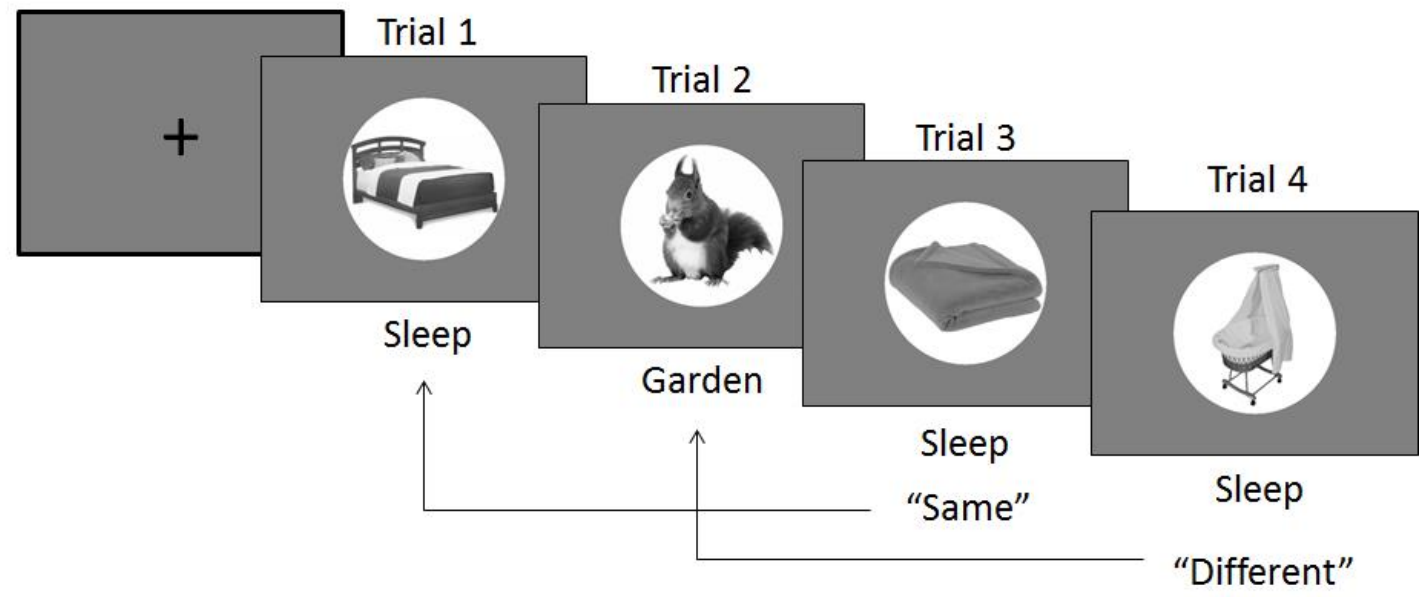

Figure 1

Example trials for the 1-back (Panel A) and 2-back (Panel B) tasks. 
(A)

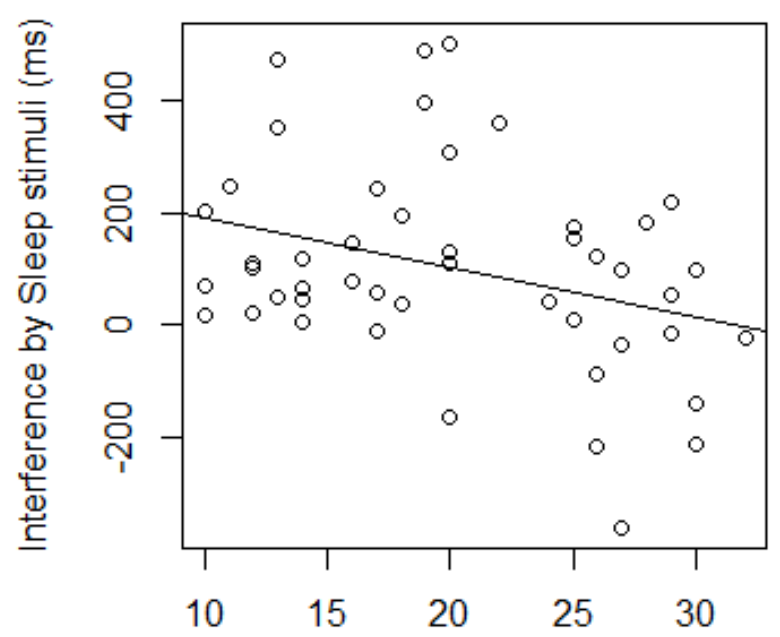

Pre-sleep Cognitive Arousal

(B)

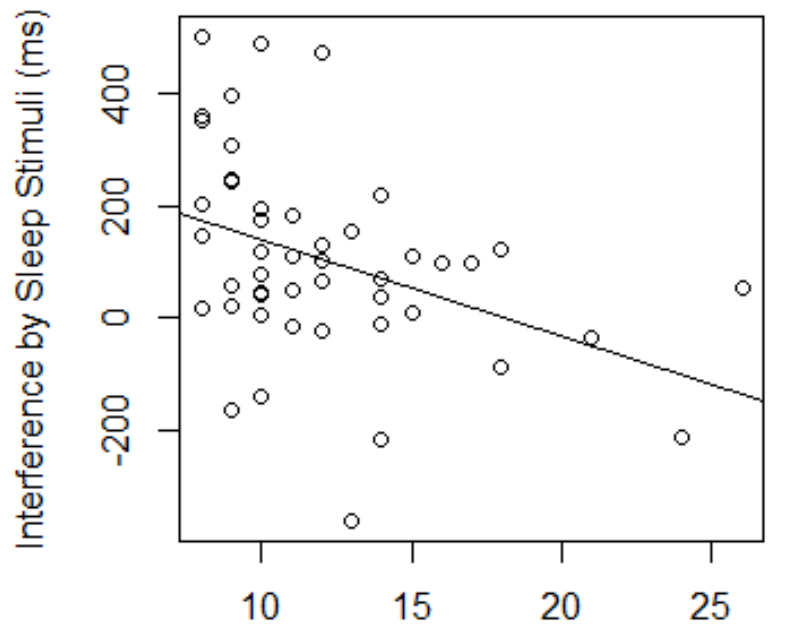

Pre-sleep Somatic Arousal

Figure 2

Interference by sleep stimuli in the 2-back task as a function of pre-sleep cognitive arousal (Panel A) and somatic arousal (Panel B). Plotted values are jittered by random noise. 


\section{Appendix}

Sleep-related and control stimuli that were used in the dot-probe task are presented in Table A1 with the scores of the valence and arousal, which were rated on a 7-point scale $(1=$ very negative $/$ unpleasant and $7=$ very positive $/$ pleasant; $1=$ very passive $/$ calm to $7=$ very active / aroused). The valence and arousal scores were well correlated between the sleep and control words ( $r=.71$ for valence, $r=.61$ for arousal), indicating that negative sleep words were paired with negative non-sleep words.

Table A1.

Sleep-related and Non-sleep-related Stimuli Presented in the Dot-probe Task (with Valence and Arousal Scores)

\begin{tabular}{|l|c|c|l|c|c|}
\hline Sleep words & Valence & Arousal & Neutral words & Valence & Arousal \\
\hline $\begin{array}{l}\text { Hoofdkussen } \\
\text { (pillow) }\end{array}$ & 3.90 & 3.40 & $\begin{array}{l}\text { Vrachtwagen } \\
\text { (truck) }\end{array}$ & 3.80 & 4.50 \\
\hline $\begin{array}{l}\text { Uitgeput } \\
\text { (exhausted) }\end{array}$ & 2.41 & 2.47 & Beledigd & 2.38 & 2.53 \\
\hline $\begin{array}{l}\text { Rusten } \\
\text { (to rest) }\end{array}$ & 5.34 & 1.89 & Vissen & & \\
\hline Droom & 5.61 & 3.77 & Schip & 4.17 & 3.69 \\
(dream) & & & (to fish) & 4.50 & 4.23 \\
\hline Bed & 5.11 & 2.34 & Vol & & \\
(bed) & & & (full) & & \\
\hline Laken & 4.25 & 2.95 & Oever & 4.45 & 2.97 \\
(sheet) & & & (bank) & & \\
\hline Wakker & 4.89 & 4.69 & Nummer & & \\
\hline
\end{tabular}




\begin{tabular}{|c|c|c|c|c|c|}
\hline (awake) & & & (number) & & \\
\hline $\begin{array}{l}\text { Geeuwen } \\
\text { (to yawn) }\end{array}$ & 3.46 & 2.94 & $\begin{array}{l}\text { Druppen } \\
\text { (to drip) }\end{array}$ & 3.57 & 3.87 \\
\hline $\begin{array}{l}\text { Doodmoe } \\
\text { (exhausted) }\end{array}$ & 2.16 & 1.75 & $\begin{array}{l}\text { Nederig } \\
\text { (humble) }\end{array}$ & 3.55 & 2.81 \\
\hline $\begin{array}{l}\text { Donker } \\
\text { (dark) }\end{array}$ & 3.34 & 3.5 & $\begin{array}{l}\text { Gebouw } \\
\text { (building) }\end{array}$ & 4.19 & 3.31 \\
\hline $\begin{array}{l}\text { Klok } \\
\text { (clock) }\end{array}$ & 4.13 & 3.54 & $\begin{array}{l}\text { Bril } \\
\text { (glasses) }\end{array}$ & 3.95 & 3.02 \\
\hline $\begin{array}{l}\text { Snurken } \\
\text { (to snore) }\end{array}$ & 2.59 & 3.87 & $\begin{array}{l}\text { bijster } \\
\text { (very) }\end{array}$ & 3.59 & 3.9 \\
\hline $\begin{array}{l}\text { Actief } \\
\text { (active) }\end{array}$ & 5.09 & 3.52 & $\begin{array}{l}\text { Zilver } \\
\text { (silver) }\end{array}$ & 5.08 & 3.50 \\
\hline $\begin{array}{l}\text { Slapen } \\
\text { (to sleep) }\end{array}$ & 5.50 & 1.91 & $\begin{array}{l}\text { Plaats } \\
\text { (place) }\end{array}$ & 4.23 & 3.22 \\
\hline $\begin{array}{l}\text { Nacht } \\
\text { (night) }\end{array}$ & 4.47 & 2.86 & $\begin{array}{l}\text { Water } \\
\text { (water) }\end{array}$ & 4.95 & 3.78 \\
\hline $\begin{array}{l}\text { Wekken } \\
\text { (to arouse) }\end{array}$ & 3.66 & 4.84 & $\begin{array}{l}\text { Zweren } \\
\text { (to swear) }\end{array}$ & 3.39 & 4.59 \\
\hline $\begin{array}{l}\text { Woelen } \\
\text { (to toss) }\end{array}$ & 3.23 & 5.16 & $\begin{array}{l}\text { Kuisen } \\
\text { (to clean) }\end{array}$ & 3.69 & 4.75 \\
\hline $\begin{array}{l}\text { Onrustig } \\
\text { (restless) }\end{array}$ & 2.69 & 5.25 & $\begin{array}{l}\text { Verwaand } \\
\text { (conceited) }\end{array}$ & 2.27 & 3.84 \\
\hline $\begin{array}{l}\text { Dutje } \\
\text { (nap) }\end{array}$ & 3.09 & 3.4 & $\begin{array}{l}\text { Baard } \\
\text { (beard) }\end{array}$ & 4.06 & 3.17 \\
\hline $\begin{array}{l}\text { Vermoeiend } \\
\text { (tiresome) }\end{array}$ & 2.63 & 4.11 & $\begin{array}{l}\text { Autoriteit } \\
\text { (authority) }\end{array}$ & 3.52 & 4.73 \\
\hline
\end{tabular}




\begin{tabular}{|l|c|c|c|c|c|}
\hline Mean & 3.92 & 3.41 & & 3.88 & 3.58 \\
\hline SD & 1.10 & 1.05 & & 0.70 & 0.72 \\
\hline
\end{tabular}

